\title{
Accurate nonrelativistic ground-state energies of $3 d$ transition metal atoms
}

Cite as: J. Chem. Phys. 141, 244110 (2014); https://doi.org/10.1063/1.4903985

Submitted: 11 September 2014 . Accepted: 01 December 2014 . Published Online: 30 December 2014

A. Scemama, T. Applencourt (D), E. Giner, and M. Caffarel (D)
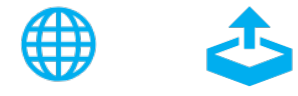

\section{ARTICLES YOU MAY BE INTERESTED IN}

A deterministic alternative to the full configuration interaction quantum Monte Carlo method

The Journal of Chemical Physics 145, 044112 (2016); https://doi.org/10.1063/1.4955109

Iterative perturbation calculations of ground and excited state energies from multiconfigurational zeroth-order wavefunctions

The Journal of Chemical Physics 58, 5745 (1973); https://doi.org/10.1063/1.1679199

Gaussian basis sets for use in correlated molecular calculations. I. The atoms boron through neon and hydrogen

The Journal of Chemical Physics 90, 1007 (1989); https://doi.org/10.1063/1.456153

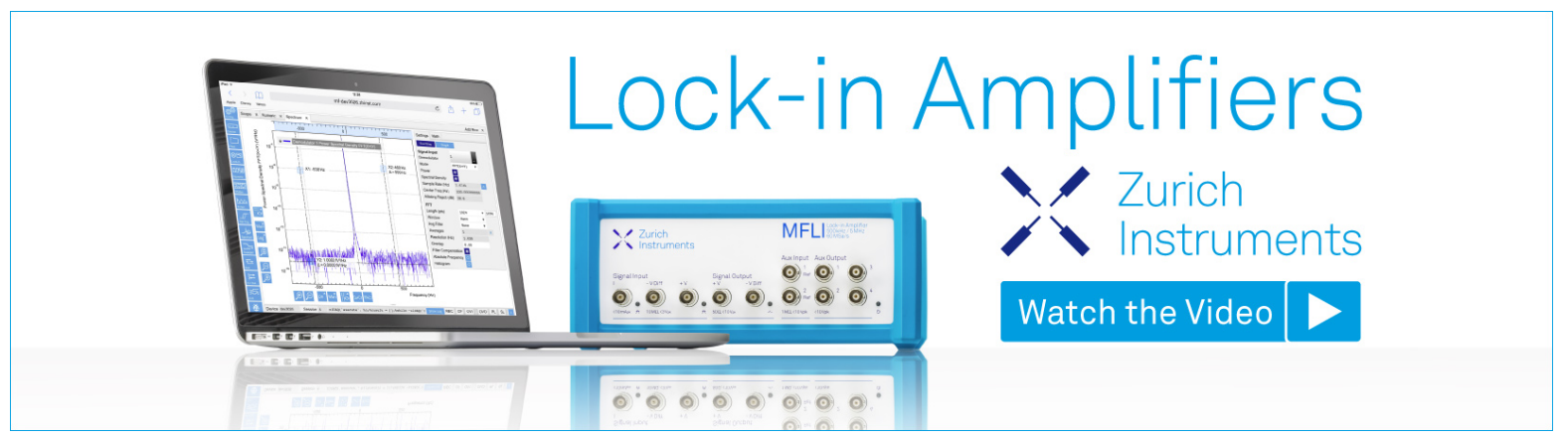




\title{
Accurate nonrelativistic ground-state energies of $3 d$ transition metal atoms
}

\author{
A. Scemama, T. Applencourt, E. Giner, and M. Caffarel \\ Lab. Chimie et Physique Quantiques, CNRS-Université de Toulouse, Toulouse, France
}

(Received 11 September 2014; accepted 1 December 2014; published online 30 December 2014)

\begin{abstract}
We present accurate nonrelativistic ground-state energies of the transition metal atoms of the $3 d$ series calculated with Fixed-Node Diffusion Monte Carlo (FN-DMC). Selected multi-determinantal expansions obtained with the CIPSI (Configuration Interaction using a Perturbative Selection made Iteratively) method and including the most prominent determinants of the full configuration interaction expansion are used as trial wavefunctions. Using a maximum of a few tens of thousands determinants, fixed-node errors on total DMC energies are found to be greatly reduced for some atoms with respect to those obtained with Hartree-Fock nodes. To the best of our knowledge, the FN-DMC/(CIPSI nodes) ground-state energies presented here are the lowest variational total energies reported so far. They differ from the recently recommended non-variational values of McCarthy and Thakkar [J. Chem. Phys. 136, 054107 (2012)] only by a few percents of the correlation energy. Thanks to the variational property of FN-DMC total energies, our results provide exact lower bounds for the absolute value of all-electron correlation energies, $\left|E_{c}\right|$. (C) 2014 AIP Publishing LLC. [http://dx.doi.org/10.1063/1.4903985]
\end{abstract}

\section{INTRODUCTION}

An accurate knowledge of nonrelativistic ground-state energies of atoms is known to be of great interest for computational chemistry. Atomic total energies are indeed routinely used to calibrate theoretical studies in electronic structure theory. For example, let us cite the search for more accurate exchange-correlation energy functionals in Density Functional Theory (DFT), the calibration of various approximations in wavefunction-based approaches (finite basis set effects, truncation at a given order in multi-particle excitations, etc.), the study of the fixed-node approximation in quantum Monte Carlo (QMC), the definition of alternative/exotic electronic approaches, etc. Furthermore, by combining experimental results and accurate nonrelativistic values, some valuable information about relativistic effects can also be obtained.

Here, accurate nonrelativistic, clamped nucleus, allelectron ground-state energies for the metal atoms of the $3 d$ series (from $\mathrm{Sc}$ to $\mathrm{Zn}$ ) are reported. Calculations are performed using the Fixed-Node Diffusion Monte Carlo (FN-DMC) approach, a QMC method known to be particularly powerful for computing ground-state energies. ${ }^{1,2}$ An overwhelming number of works have been devoted to the calculation of accurate atomic ground-state energies using various highly correlated approaches; here, we shall only restrict ourselves to give the typical accuracies presently achievable. For small atoms (say, less than 10 electrons, that is, from $\mathrm{H}$ to $\mathrm{Ne}$ for neutral atoms), very accurate values with errors smaller than $10^{-4}-10^{-5}$ a.u. (or much smaller for the lightest atoms) can be obtained. For heavier atoms up to Ar (18 electrons), the accuracy reduces to the millihartree level ( $\sim$ chemical accuracy). For even bigger atoms to obtain a precision close to the millihartree is problematic and only a small number of results have been published. Regarding quantum Monte Carlo studies using FN-DMC or a closely related QMC variant, most of the works have been concerned with atoms from $\mathrm{Li}$ to $\mathrm{Ne}$; for the most recent ones, see, e.g., Refs. 3-6. For heavier atoms, most calculations have been performed using pseudo-potentials to remove core electrons (see, e.g., Refs. 7-9). At the all-electron level, very little has been done. We can essentially cite the FN-DMC calculations by Ma et al. ${ }^{10}$ for the $\mathrm{Ar}, \mathrm{Kr}$, and $\mathrm{Xe}$ atoms; calculations for the $\mathrm{Cu}$ atom and its cation; ${ }^{11,12}$ and two studies by Buendia and collaborators for $3 d$ transition metal atoms. ${ }^{4,6}$

It is fair to say that FN-DMC is presently the most accurate method for computing total ground state energies for large enough electronic systems. Potentially, diffusion Monte Carlo allows an exact stochastic solution of the Schrödinger equation. Several sources of error make, in practice, FN-DMC simulations non-exact. However, most of the errors are not of a fundamental nature and can be easily kept under control (mainly, the statistical, finite time-step, and population control errors). In contrast, the fixed-node error resulting from the use of trial wavefunctions with approximate nodes is much more problematic since, up to now, no simple and systematic scheme to control this error has been devised. Note that the fixed-node approximation is variational, $E_{\mathrm{FN}} \geq E_{0}$, a convenient property to get upper and lower bounds for total energies and absolute values of correlation energies, respectively [in contrast, e.g., with the non-variational character of the commonly used Coupled Cluster with Singles, Doubles and Triples perturbatively (CCSD(T)) or Møller-Plesset approaches].

To decrease the fixed-node error, the common strategy is to use high-quality trial wavefunctions and to resort to multi-parameter stochastic optimization techniques to get the best parameters (usually via minimization of the total energy and/or its variance). A great variety of functional forms 
have thus been introduced for the wavefunction (see, e.g., Refs. 12-22), and various optimization techniques designed to be efficient in a Monte Carlo context have been developed (e.g., Ref. 23).

In this work, we shall employ a different strategy based on the use of a new class of trial wavefunctions very recently introduced in QMC simulations. ${ }^{24}$ The key ideas are (i) to avoid the use of a Jastrow factor, (ii) to rely on standard multi-determinant configuration interaction (CI) expansions, and (iii) to avoid any stochastic optimization of the wavefunction. To build CI expansions compact enough to make QMC feasible in practice, we employ a selected CI method (CIPSI (Configuration Interaction using a Perturbative Selection made Iteratively) algorithm, see Refs. 26 and 32) allowing to extract the most prominent determinants of the Full Configuration Interaction (FCI) expansion. Using an efficient implementation of the calculation of the multideterminant part ${ }^{34,35}$ expansions containing a large number of determinants can be used in QMC. In the present application to $3 d$ atoms, expansions up to a few tens of thousands of determinants have been employed. A nice feature of using CIPSI wavefunctions is that simulations can be performed in a fully black-box way: The CI program is first run, then the QMC code is started using as input the CI wavefunction directly as it comes from the output of the CI program. In particular, the preliminary multi-parameter Monte Carlo optimization of the trial wavefunction done in standard FNDMC approaches (see, e.g., Ref. 36) is avoided. A number of recent applications ${ }^{24,37,38}$ have illustrated that CIPSI trial wavefunctions are effective in improving nodes. Furthermore, the fixed-node energy is found to decrease monotonically as a function of the number of determinants or the size of the basis set. This remarkable property allows to define a simple control of the fixed-node error. The price to pay for such attractive properties is that Jastrow-free CI expansions have (much) larger variances and thus require longer simulation times. However, this aspect is in practice not as critical as it may appear. By using our efficient implementation of the calculation of the multi-determinant part and by running our QMC code on medium size computational platforms with a few thousands of compute cores, it has been possible to realize the entire set of calculations presented here for the $3 d$ series in less than two days. From a more general perspective, we would like to emphasize that the possibility of having a simple, automatic, and systematic way of decreasing and controlling the fixed-node bias is of utmost practical importance. It clearly remains true even at the price of more central processing unit-intensive simulations that will anyway become more and more easy to perform in view of the embarrassingly parallel property of QMC and the rapid development of ever more powerful parallel machines.

The FN-DMC/(CIPSI nodes) total ground-state energies of metal atoms of the $3 d$ series obtained here are compared to the very recent results of Buendia et al. ${ }^{6}$ using a FN-DMCtype approach with a single-determinant trial wavefunction. A systematic improvement in FN total energies is obtained for all atoms, except for $\mathrm{Zn}$ for which similar results are found. For the lightest $3 d$ atoms, the total energy values are much improved (a gain up to about $4.5 \times 10^{-2}$ a.u. for Sc).
To the best of our knowledge, the data presented here are the lowest variational total ground-state energies reported so far for the $3 d$ transition metal atoms. They differ from the recently recommended non-variational values of McCarthy and Thakkar $^{39}$ (denoted as McCT in what follows) obtained by an hybrid approach combining MP2 results at completebasis-set (CBS) limit and accurate CCSD(T) values, only by a few percents of the correlation energy, namely, between $5.6 \%$ and $8 \%$ while these authors estimate their errors to be about $\pm 3 \%$. Thanks to the variational property of FN-DMC total energies, our results also provide exact lower bounds for the absolute value of all-electron correlation energies, $\left|E_{c}\right|$.

\section{METHODS AND COMPUTATIONAL DETAILS}

\section{A. CIPSI}

The CIPSI method, and similar approaches closely related, have been introduced and developed a long time ago by a number of authors (see Refs. 25-33). In a few words, the approach consists in building the multi-determinantal expansion iteratively by selecting at each step one determinant (or a group of determinants) according to a perturbative criterion. A determinant $D_{i}$ (or a group of determinants) is added to the current wavefunction if its (their) energetic contribution(s) calculated by second-order perturbation theory is (are) sufficiently large. In this way, the wavefunction is built hierarchically, the most important determinants of the FCI solution entering first in the expansion. Such a construction must be contrasted with standard approaches (configuration interaction with singles, configuration interaction with singles and doubles, etc.), where the contributions at a given order are calculated by considering all possible particleexcitations with respect to some reference wavefunction (usually, the Hartree-Fock (HF) solution). The CIPSI multideterminantal expansion is thus much more compact than standard expansions, an important practical point for $\mathrm{FN}$ DMC where the trial wavefunction and its derivatives must be computed a very large number of times during the simulations. Let us now briefly summarize the algorithm. More details can be found in Ref. 24 and references therein.

In multi-determinantal expansions, the ground-state wavefunction $\left|\Psi_{0}\right\rangle$ is written as a linear combination of Slater determinants $\left\{\left|D_{i}\right\rangle\right\}$, each determinant corresponding to a given occupation by the $N_{\alpha}$ and $N_{\beta}$ electrons of $N=N_{\alpha}+N_{\beta}$ electrons among a set of $M$ spin-orbitals $\left\{\phi_{1}, \ldots, \phi_{M}\right\}$ (restricted case). The best representation of the exact wavefunction in the entire determinantal basis is the FCI wavefunction written as

$$
\left|\Psi_{0}\right\rangle=\sum_{i} c_{i}\left|D_{i}\right\rangle,
$$

where $c_{i}$ are the ground-state coefficients obtained by diagonalizing the Hamiltonian matrix, $H_{i j}=\left\langle D_{i}|H| D_{j}\right\rangle$, within the orthonormalized set, $\left\langle D_{i} \mid D_{j}\right\rangle=\delta_{i j}$, of determinants $\left|D_{i}\right\rangle$.

In its simplest form, the multi-determinant wavefunction is iteratively built as follows. Let us call $\left|\Psi_{0}^{(n)}\right\rangle=\sum_{i \in S_{n}} c_{i}^{(n)}\left|D_{i}\right\rangle$ the current wavefunction at iteration $n$, where $S_{n}$ is the set 
of selected determinants at iteration $n$. Typically, at the initial step $n=0$, a mono-determinantal HF-type or a short completeactive-space-self-consistent-field-type wavefunction is used. The first step consists in collecting all different determinants $\left|D_{i_{c}}\right\rangle$ connected by $H$ to $\left|\Psi_{0}^{(n)}\right\rangle$, that is, $\left\langle\Psi_{0}^{(n)}|H| D_{i_{c}}\right\rangle \neq 0$. Then, the second-order correction to the total energy resulting from each connected determinant is computed,

$$
\delta e\left(\left|D_{i_{c}}\right\rangle\right)=-\frac{\left\langle\Psi_{0}^{(n)}|H| D_{i_{c}}\right\rangle^{2}}{\left\langle D_{i_{c}}|H| D_{i_{c}}\right\rangle-E_{0}^{(n)}}
$$

and the determinant (or group of determinants) $\left|D_{i_{c}^{*}}\right\rangle$ associated with the largest $|\delta e|$ (or greater than a given threshold) is (are) added to the reference subspace

$$
S_{n} \rightarrow S_{n+1}=S_{n} \cup\left\{\left|D_{i_{c}^{*}}\right\rangle\right\} .
$$

Finally, the Hamiltonian matrix is then diagonalized within $S_{n+1}$ to obtain the new wavefunction at iteration $n+1$ and the process is iterated until a target size $N_{\text {dets }}$ for the reference subspace is reached. The CIPSI wavefunction issued from this selection process is the trial wavefunction used here for FN-DMC.

\section{B. FN-DMC}

For a detailed presentation of the theoretical and practical aspects of FN-DMC, the reader is referred to the literature, e.g., Ref. 40. Here, let us just emphasize that the central quantity of such approaches is the trial wavefunction $\Psi_{T}$ determining both the magnitude of the fixed-node error through its approximate nodes and the quality of the statistical convergence (good trial wavefunctions imply small statistical fluctuations). The computational cost of FN-DMC is almost entirely determined by the evaluation at each Monte Carlo step of the value of $\Psi_{T}$ and its first (drift vector) and second derivatives (Laplacian needed for the local energy). In view of the very large number of MC steps usually required (typically at least billions and often much more), it is essential to compute such quantities very rapidly. In the present work, the typical size of the expansion considered is a few tens of thousands of determinants. Some care is thus required when computing such expansions to keep the computational cost reasonable. The various aspects regarding this problem are presented in Ref. 35 .

\section{Computational details}

The atomic basis sets used for the calculations are the Slater-type orbitals of Bunge ${ }^{41}$ supplemented with four additional $4 f$ and three $5 g$ functions (a total of 112 atomic basis functions). All the CIPSI calculations have been performed using Hartree-Fock molecular orbitals using the code developed in our group (quantum package), and all the FN-DMC calculations have been performed using QMC $=$ Chem. $^{42}$

For each atom, the CIPSI calculation was stopped when more than $10^{6}$ determinants were selected in the variational wave function. This wave function was then truncated such that the least significant determinants contributing to $0.5 \%$ of the norm of the wave function were discarded: $10^{4}-5 \times 10^{4}$ determinants were kept. This wave function was used without any modification as the trial wave function for the FN-DMC calculations (no Jastrow factor).

For the FN-DMC calculations, we have employed the algorithm described in Ref. 43 allowing us to use a small constant number of walkers. A block consisted of 30 walkers performing 5000 steps with a time step of $10^{-5}$ a.u., a value chosen such that the time-step error was smaller than the statistical error. Long enough simulations have been performed to make the statistical error negligible with respect to the fixed-node one: depending on the atom, a number of blocks between $7 \times 10^{4}$ and $1.5 \times 10^{5}$ were calculated $\left(\sim 10^{10} \mathrm{MC}\right.$ steps $)$.

\section{RESULTS}

In Table I, the variational energy, the number of determinants in the CIPSI expansion, an estimate of the percentage of the total CE recovered, and the average value of the $S^{2}$ total spin operator for each trial wavefunction $\Psi_{T}$ used in FN-DMC are given. The CE's reported are calculated from the recommended values of McCarthy and Thakkar. ${ }^{39}$ We emphasize that, in contrast with the present work, these values have not been computed directly from a unique (very) accurate energy calculation but have been obtained indirectly by combining Møller-Plesset (MP2) correlation energies extrapolated at the CBS limit and $\operatorname{CCSD}(\mathrm{T})$ calculations using Dunning's basis sets of various sizes. The percentages of correlation energies retrieved at the CIPSI variational level are found to be around $60 \%$. It is important to note that the correlation effects recovered in such CI calculations are mostly valence correlation effects (valence basis set) and non-dynamical correlation effects resulting from the use of multi-determinant expansions including the most prominent determinants. In particular, most of the energetic core contributions are not taken into account in these valence CI calculations. Some caution is thus needed when comparing the total energies obtained with the Slater-Jastrow wavefunctions usually employed in FN-DMC and the valence CI wavefunctions proposed here. Finally, note that the total

TABLE I. Number of determinants, $\mathrm{N}_{\text {dets }}$, of the CIPSI expansion used as FN-DMC trial wavefunction. Variational energy, $\mathrm{E}_{\mathrm{var}}$ (CIPSI), estimated percentages of the correlation energy (CE) recovered, and eigenvalue of the $S^{2}$ spin operator. Energy in hartree.

\begin{tabular}{lcccc}
\hline \hline Atom & $\mathrm{N}_{\text {dets }}$ & $\mathrm{E}_{\mathrm{var}}(\mathrm{CIPSI})$ & $(\mathrm{CE}$ in $\%)$ & $\mathrm{S}^{2}$ \\
\hline $\mathrm{Sc}^{2} \mathrm{D}\left(\mathrm{d}^{1} \mathrm{~s}^{2}\right)$ & 11389 & -760.32556 & $(66.5)$ & 0.75 \\
$\mathrm{Ti}^{3} \mathrm{~F}\left(\mathrm{~d}^{2} \mathrm{~s}^{2}\right)$ & 14054 & -849.02624 & $(66.9)$ & 1.99 \\
$\mathrm{~V}^{4} \mathrm{~F}\left(\mathrm{~d}^{3} \mathrm{~s}^{2}\right)$ & 12441 & -943.53667 & $(64.9)$ & 3.74 \\
$\mathrm{Cr}^{7} \mathrm{~S}\left(\mathrm{~d}^{5} \mathrm{~s}^{1}\right)$ & 10630 & -1044.03692 & $(63.6)$ & 11.97 \\
$\mathrm{Mn}{ }^{6} \mathrm{~S}\left(\mathrm{~d}^{5} \mathrm{~s}^{2}\right)$ & 11688 & -1150.57902 & $(63.0)$ & 8.73 \\
$\mathrm{Fe}{ }^{5} \mathrm{D}\left(\mathrm{d}^{6} \mathrm{~s}^{2}\right)$ & 13171 & -1263.21805 & $(62.5)$ & 5.99 \\
$\mathrm{Co}^{4} \mathrm{~F}\left(\mathrm{~d}^{7} \mathrm{~s}^{2}\right)$ & 15949 & -1382.24964 & $(62.8)$ & 3.74 \\
$\mathrm{Ni}{ }^{3} \mathrm{~F}\left(\mathrm{~d}^{8} \mathrm{~s}^{2}\right)$ & 15710 & -1507.74694 & $(62.3)$ & 1.99 \\
$\mathrm{Cu}{ }^{2} \mathrm{~S}\left(\mathrm{~d}^{10} \mathrm{~s}^{1}\right)$ & 48347 & -1639.96605 & $(63.3)$ & 0.75 \\
$\mathrm{Zn}{ }^{1} \mathrm{~S}\left(\mathrm{~d}^{10} \mathrm{~s}^{2}\right)$ & 44206 & -1778.82784 & $(60.5)$ & 0.00 \\
\hline \hline
\end{tabular}


TABLE II. FN-DMC total energies for the $3 d$ series of transition metal atoms together with the percentage of correlation energy recovered for different nodal structures. Energy in hartree.

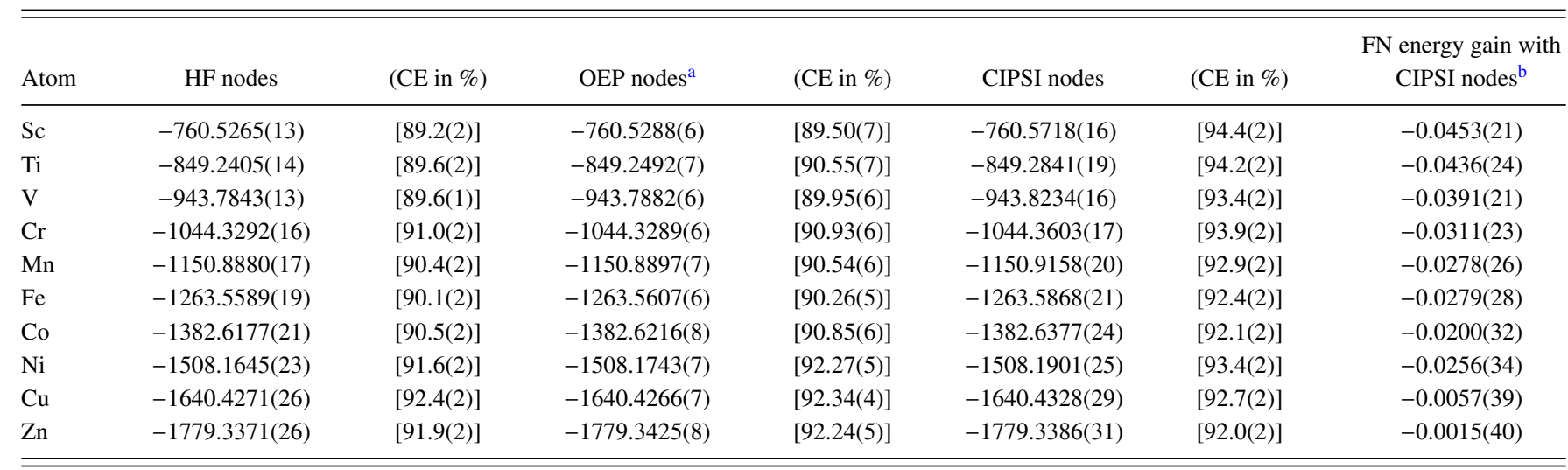

${ }^{\mathrm{a}}$ Reference 6.

${ }^{\mathrm{b}}$ Difference between FN-DMC energies obtained with HF nodes (column 1) and newly proposed CIPSI nodes (column 5).

spin values $S^{2}$ obtained from the truncated CIPSI expansions show that the trial wavefunctions built are almost pure spineigenstates.

In Table II, the fixed-node DMC total energies obtained using standard Hartree-Fock nodes and newly proposed CIPSI nodes are reported. For the sake of comparison, we also give the very recent results of Buendia et al. ${ }^{6}$ that were up to now the lowest variational fixed-node energies reported for these atoms. In their study, the trial wavefunctions employed are written as the product of a nodeless correlation factor and a so-called model function obtained within the parametrized Optimized Effective Potential (OEP) approximation. The model function determining the nodal structure is written as a linear combination of a few Configuration State Functions (CSFs), mainly to take into account $4 s-4 p$ near-degeneracy effects. For the $\mathrm{Cr}$ and $\mathrm{Cu}$ atoms with a singly occupied $4 s$ shell, the model function is represented by a single CSF, while for the other atoms $4 s^{2} 3 d^{n}$ and $4 p^{2} 3 d^{n}$ configurations are mixed. For each type of nodes used, an estimate of the percentage of the correlation energy is also reported. The percentages retrieved by all FN-DMC calculations presented are important and range between $89 \%$ and $94 \%$. A first observation is that energies resulting from HF and OEP nodes are of comparable quality, while CIPSI nodes may lead to significantly lower fixed-node energies. The gain in energy with the new nodes is found to decrease almost uniformly with $Z$. For the lightest elements ( $\mathrm{Sc}, \mathrm{V}$, and $\mathrm{Ti}$ ), a maximum gain of about 0.045 a.u. is achieved; for the intermediate atoms $(\mathrm{Cr}-\mathrm{Ni})$, about $0.02-0.03$ a.u. is obtained, while for the two heaviest elements $(\mathrm{Cu}$ and $\mathrm{Zn})$, no gain is observed within statistical fluctuations. The fact that CIPSI performs better for lightest elements is not surprising since Hartree-Fock nodes are known to be well-adapted to atoms with spherical symmetry. In the extreme case of the $\mathrm{Cu}$ and $\mathrm{Zn}$ atoms having a totally filled and spherically symmetric $3 d$ shell, HF and CIPSI nodes give similar results. In the opposite case of light atoms, the CIPSI wavefunctions, that have many more degrees of freedom than the single-configuration HF solution to describe non-symmetrical electronic configurations, lead to much improved results. In Table III, the correlation energies resulting from our FN-DMC simulations are reported and compared to the recommended values of McCT. As already noted, these latter results have been obtained with a mixed approach including MP2-CBS and $\operatorname{CCSD}(\mathrm{T})$ calculations. According to the authors, the errors in these values are estimated to be $\pm 3 \%$. The relative differences between FNDMC/[HF nodes] or FN-DMC/[OEP nodes] and the McCT values go from $8 \%$ to $11 \%$. Using CIPSI nodes, the differences are reduced and range between $6 \%$ and $8 \%$. Note that the typical statistical error on these percentages is small and about $0.2 \%$. Although our final values for correlation energies are slightly less accurate than the estimates made by McCT, we would like to emphasize and conclude on three important points: (i) In contrast with what has been done by $\mathrm{McCT}$, our correlation energies have been directly computed with a unique highly correlated electronic structure method. No hybrid scheme mixing results of two different approaches has been employed. To the best of our knowledge, the FNDMC values presented here are the most accurate (lowest) nonrelativistic total energies ever reported for the $3 d$ transition metal atoms. (ii) As a consequence of the variational property of FN-DMC total energies and also in contrast with McCT's results, the absolute values of our correlation energies are exact lower bounds of the unknown CE's. (iii) Finally, in view

TABLE III. Fixed-node DMC correlation energies, $-E_{c}$, in hartree using HF and CIPSI nodes. Comparison with the recommended values of McCarthy and Thakkar (McCT). ${ }^{39}$

\begin{tabular}{lllll}
\hline \hline Atom & HF nodes & OEP nodes $^{\mathrm{a}}$ & CIPSI nodes & McCT $^{\mathrm{b}}$ \\
\hline $\mathrm{Sc}$ & $0.7900(13)$ & $0.7923(6)$ & $0.8353(16)$ & 0.8853 \\
$\mathrm{Ti}$ & $0.8454(14)$ & $0.8541(7)$ & $0.8890(19)$ & 0.9433 \\
$\mathrm{~V}$ & $0.9000(13)$ & $0.9039(6)$ & $0.9390(16)$ & 1.0049 \\
$\mathrm{Cr}$ & $0.9728(16)$ & $0.9725(6)$ & $1.0039(17)$ & 1.0695 \\
$\mathrm{Mn}$ & $1.0218(17)$ & $1.0235(7)$ & $1.0495(20)$ & 1.1304 \\
$\mathrm{Fe}$ & $1.1122(19)$ & $1.1140(6)$ & $1.1401(21)$ & 1.2343 \\
$\mathrm{Co}$ & $1.2016(21)$ & $1.2055(8)$ & $1.2216(24)$ & 1.3270 \\
$\mathrm{Ni}$ & $1.3043(23)$ & $1.3141(7)$ & $1.3299(25)$ & 1.4242 \\
$\mathrm{Cu}$ & $1.4634(26)$ & $1.4629(7)$ & $1.4691(29)$ & 1.5842 \\
$\mathrm{Zn}$ & $1.4890(26)$ & $1.4944(8)$ & $1.4905(31)$ & 1.6202 \\
\hline \hline
\end{tabular}

${ }^{\text {a Reference } 6 .}$

${ }^{\mathrm{b}}$ Reference 39 . 
of the great versatility of FN-DMC/CIPSI, there is no reason why improved lower bounds would not be achieved in the near future.

\section{ACKNOWLEDGMENTS}

A.S. and M.C. thank the Agence Nationale pour la Recherche (ANR) for support through Grant No. ANR 2011 BS08 004 01. This work has been possible thanks to the computational support of CALMIP through a Meso-Challenge on their new Eos supercomputer (www.calmip.univtoulouse.fr).

${ }^{1}$ B. L. Hammond, W. A. Lester, Jr., and P. J. Reynolds, Monte Carlo Methods in Ab Initio Quantum Chemistry, World Scientific Lecture and Course Notes in Chemistry (World Scientific, 1994), Vol. 1.

${ }^{2}$ W. M. C. Foulkes, L. Mitas, R. J. Needs, and G. Rajagopal, Rev. Mod. Phys. 73, 33 (2001)

${ }^{3}$ M. D. Brown, J. R. Trail, P. L. Rios, and R. J. Needs, J. Chem. Phys. 126, 224110 (2007).

${ }^{4}$ A. Sarsa, E. Buendia, F. J. Galvez, and P. Maldonado, J. Phys. Chem. A 112, 2074 (2008).

${ }^{5}$ P. L. Rios, P. Seth, N. D. Drummond, and R. J. Needs, Phys. Rev. E 86, 036703 (2012).

${ }^{6}$ E. Buendia, F. J. Galvez, P. Maldonado, and A. Sarsa, Chem. Phys. Lett. 559, 12 (2013).

${ }^{7}$ L. Mitas, Phys. Rev. A 49, 4411 (1994).

${ }^{8}$ L. Wagner and L. Mitas, Chem. Phys. Lett. 370, 412 (2003).

${ }^{9}$ S. Sokolova and A. Luchow, Chem. Phys. Lett. 320, 421 (2000).

${ }^{10}$ A. Ma, N. D. Drummond, M. D. Towler, and R. J. Needs, Phys. Rev. E 71, 066704 (2005).

${ }^{11}$ M. Caffarel, J. P. Daudey, J. L. Heully, and A. Ramirez-Solis, J. Chem. Phys. 123, 094102 (2005).

${ }^{12}$ T. Bouabça, B. Braîda, and M. Caffarel, J. Chem. Phys. 133, 044111 (2010).

${ }^{13}$ K. E. Schmidt and J. W. Moskowitz, J. Chem. Phys. 93, 4172 (1990).

${ }^{14}$ H. J. Flad, M. Caffarel, and A. Savin, "Quantum Monte Carlo Calculations with Multi-Reference Trial Wave Functions," in Recent Advances in Quantum Monte Carlo Methods (World Scientific Publishing, 1997).

${ }^{15}$ C. Filippi and C. J. Umrigar, J. Chem. Phys. 105, 213 (1996).

${ }^{16}$ B. Braiida, J. Toulouse, M. Caffarel, and C. J. Umrigar, J. Chem. Phys. 134, 0184108 (2011).

${ }^{17}$ A. G. Anderson and W. A. Goddard III, J. Chem. Phys. 132, 164110 (2010).

${ }^{18}$ F. Fracchia, C. Filippi, and C. Amovilli, J. Chem. Theory Comput. 8, 1943 (2012).
${ }^{19}$ A. Monari, A. Scemama, and M. Caffarel, "Large-scale quantum Monte Carlo electronic structure calculations on the egee grid," in Remote Instrumentation for eScience and Related Aspects, edited by F. Davoli, M. Lawenda, N. Meyer, R. Pugliese, J. Wglarz, and S. Zappatore (Springer, New York, 2012), pp. 195-207.

${ }^{20}$ M. Bajdich, L. Mitáš, G. Drobnỳ, and L. K. Wagner, Phys. Rev. Lett. 96, 130201 (2006).

${ }^{21}$ M. Casula, C. Attaccalite, and S. Sorella, J. Chem. Phys. 121, 7110 (2004).

${ }^{22}$ P. L. Rios, A. Ma, N. D. Drummond, M. D. Towler, and R. J. Needs, Phys. Rev. E 74, 066701 (2006).

${ }^{23}$ C. J. Umrigar, J. Toulouse, C. Filippi, S. Sorella, and R. G. Hennig, Phys. Rev. Lett. 98, 110201 (2007).

${ }^{24}$ E. Giner, A. Scemama, and M. Caffarel, Can. J. Chem. 91, 879 (2013).

${ }^{25}$ C. F. Bender and E. R. Davidson, Phys. Rev. 183, 23 (1969).

${ }^{26}$ B. Huron, P. Rancurel, and J. P. Malrieu, J. Chem. Phys. 58, 5745 (1973).

${ }^{27}$ R. J. Buenker and S. D. Peyerimhoff, Theor. Chim. Acta 35, 33 (1974).

${ }^{28}$ R. J. Buenker and S. D. Peyerimhoff, Theor. Chim. Acta 39, 217 (1975).

${ }^{29}$ R. J. Buenker, S. D. Peyerimhoff, and W. Butscher, Mol. Phys. 35, 771 (1978).

${ }^{30}$ P. J. Bruna, D. S. Peyerimhoff, and R. J. Buenker, Chem. Phys. Lett. 72, 278 (1980).

${ }^{31}$ R. J. Buenker, S. D. Peyerimhoff, and P. J. Bruna, Computational Theoretical Organic Chemistry (Reidel, Dordrecht, 1981).

${ }^{32}$ S. Evangelisti, J. P. Daudey, and J. P. Malrieu, Chem. Phys. 75, 91 (1983).

${ }^{33}$ R. J. Harrison, J. Chem. Phys. 94, 5021 (1991).

${ }^{34}$ A. Scemama, M. Caffarel, E. Oseret, and W. Jalby, Lect. Notes Comput. Sci. 7851, 118-127 (2013).

${ }^{35}$ A. Scemama, M. Caffarel, E. Oseret, and W. Jalby, J. Comput. Chem. 34, 938 (2013).

36. Toulouse and C. J. Umrigar, J. Chem. Phys. 128, 174101 (2008).

${ }^{37}$ E. Giner, A. Scemama, and M. Caffarel, "Fixed-node diffusion Monte Carlo potential energy curve of the fluorine molecule $F_{2}$ using selected configuration interaction trial wavefunctions," J. Chem. Phys. (submitted); preprint arXiv:1409.3671.

${ }^{38}$ E. Giner, "Méthodes d'interaction de configurations et Monte Carlo quantique : Marier le meilleur des deux mondes (configuration interaction and QMC: The best of both worlds)," Ph.D. thesis (University of Toulouse, 2014), https://hal.archives-ouvertes.fr/tel-01077016.

${ }^{39}$ S. P. McCarthy and A. J. Thakkar, J. Chem. Phys. 136, 054107 (2012).

${ }^{40} \mathrm{M}$. Caffarel, Quantum Monte Carlo Methods in Chemistry, Encyclopedia of Applied and Computational Mathematics (Springer, 2012).

${ }^{41}$ C. F. Bunge, J. A. Barrientos, and A. Bunge-Vivier, At. Data Nucl. Data Tables 53(1), 113-162 (1993).

${ }^{42}$ See http://qmechem.ups-tlse.fr for "Quantum Monte Carlo for Chemistry @Toulouse."

${ }^{43}$ R. Assaraf, M. Caffarel, and A. Khelif, Phys. Rev. E 61(4), 4566-4575 (2000). 